\title{
ON RIGIDITY OF GENERALIZED CONFORMAL STRUCTURES
}

\author{
SAMIR BEKKARA AND ABDELGHANI ZEGHIB
}

\begin{abstract}
The classical Liouville Theorem on conformal transformations determines local conformal transformations on the Euclidean space of dimension $\geq 3$. Its natural adaptation to the general framework of Riemannian structures is the 2-rigidity of conformal transformations, that is such a transformation is fully determined by its 2-jet at any point. We prove here a similar rigidity for generalized conformal structures defined by giving a one parameter family of metrics (instead of scalar multiples of a given one) on each tangent space.
\end{abstract}

\section{Contents}

1. Introduction

2. Further investigations

3. Some preliminaries

4. A generalized Braid Lemma

5. Proof of the generalized Liouville Theorem

6. Sub-rigidity of lightlike metrics, Proof of Theorem 2.2

References

\section{Introduction}

Rough notion. For a vector space $E$, let $\operatorname{Sym}(E)$ be the space of symmetric bilinear forms on $E, \operatorname{Sym}^{+}(E)$ those which are positive definite, and $\operatorname{Sym}^{*}(E)$ the nondegenerate ones.

For a manifold $M$, one defines similarly fiber bundles $\operatorname{Sym}(T M), \operatorname{Sym}^{+}(T M)$ and $\operatorname{Sym}^{*}(T M)$ associated to its tangent bundle $T M$.

A Riemannian metric is nothing but a section of $\mathrm{Sym}^{+}(T M)$. Recall on the other hand that a (Riemannian) conformal structure consists in giving a class $[g]$ of Riemannian metrics, for the conformal equivalence relation $\sim$ between metrics: $g_{1} \sim g_{2}$ if there exists a function $\sigma$ on $M$ such that $g_{1}=e^{\sigma} g_{2}$. Thus, a conformal structure consists in giving a section of the projectivized of $\mathrm{Sym}^{+}(T M)$.

Equivalently, a conformal structure consists in giving for each point $x \in M$, a half line in $\mathrm{Sym}^{+}\left(T_{x} M\right)$.

We are now going to introduce a first rough definition of generalized conformal structures (GCS for short) by associating to each $x \in M$ a (non-parameterized) curve in $\operatorname{Sym}^{+}\left(T_{x} M\right)$. Say, this consists in giving a subset $\mathcal{C} \subset \mathrm{Sym}^{+}(T M)$ such

Date: July 24, 2018. 
that the fibers of the projection $\mathcal{C} \rightarrow M$ have dimension $\leq 1$ and are non-empty. One naturally defines the image of such a structure $\mathcal{C}$ by a diffeomorphism, and an automorphism group $A u t(\mathcal{C})$. In the sequel, automorphisms will be alternatively called isometries.

Our goal is to study such objects from the point of view of being "rigid geometric structures". Roughly speaking, $d$-rigidity means that an automorphism is fully determined by its jet up to order $d$ at any point. We have here two "limit" cases, that where the $\mathcal{C}$-fibers are points (a Riemannian metric), and the other where the $\mathcal{C}$-fibers are half-lines (a conformal structure). It is known that Riemannian metrics are 1-rigid, whereas conformal structures are 2-rigid in dimension $\geq 3$; this is the essence of classical Liouville Theorem. Our generalized case here when the $\mathcal{C}$-fibers are general curves may be expected to be as rigid as the conformal case, that is one has 2-rigidity. In some sense, one naturally expects that when going from straight lines to general curves, one can not lose of rigidity because one gets more constraints on isometries.

1.0.1. A First example. Let us start by this general example which will give evidence that some topological tameness hypotheses on $\mathcal{C}$ are in order. Let $\phi^{t}$ be a flow on $M$ and $g_{0}$ any initial metric on $M$. For any $x$, give $\mathcal{C}_{x}$ as the (parameterized) curve $t \rightarrow\left(\phi_{*}^{t} g_{0}\right)_{x} \in \mathrm{Sym}^{+}\left(T_{x} M\right)$, here $\phi_{*}^{t} g_{0}$ is the image of $g_{0}$ by $\phi^{t}$.

Observe that $\phi^{t} \in \operatorname{Aut}(\mathcal{C})$. Thus, any flow gives rise to a rough GCS with a non-trivial automorphism group, which may have a strong dynamics. One can not expect for such a structure to behave as a nice geometric structure!

1.0.2. (Regular) Definition. We are now going to propose a definition of GCS which will be proved to be adapted to our rigidity hope, just by assuming that the corresponding subset $\mathcal{C}$ is a manifold.

More precisely, let us say $\mathcal{C}$ is a regular $G C S$ if $\mathcal{C}$ is a submanifold of dimension $\operatorname{dim} M+1$ in $\operatorname{Sym}^{+}(T M)$, which is transverse to the fibers $\left(\right.$ of $\left.\operatorname{Sym}^{+}(T M) \rightarrow M\right)$. Equivalently, the projection $\mathcal{C} \rightarrow M$ is a submersion and $\operatorname{dim} \mathcal{C}=\operatorname{dim} M+1$.

Each fiber $\mathcal{C}_{x}$ is thus a (non-necessarily connected) embedded 1-dimensional submanifold. In the case of a classical conformal structure, $\mathcal{C}$ is in fact a closed submanifold and it fibers over $M$.

Let us say that $\mathcal{C}$ is generic if the tangent direction of $\mathcal{C}_{x}$ at any of its points belongs to $\operatorname{Sym}^{*}\left(T_{x} M\right)$. In other words, if $\mathcal{C}_{x}$ is parameterized as a curve $t \in \mathbb{R} \rightarrow$ $c_{x}(t) \in \operatorname{Sym}\left(T_{x} M\right)$, then $c_{x}^{\prime}(t)$ is assumed to be non-degenerate. For example, classical conformal structures are generic.

1.0.3. A second example, Infinitesimally Homogeneous case. (see 3.1). Let us consider the situation where there is a 1-dimensional submanifold $\mathcal{C}_{0} \subset \operatorname{Sym}{ }^{+}\left(\mathbb{R}^{n}\right)$ such that for any $x, \mathcal{C}_{x}=A_{x}^{*}\left(\mathcal{C}_{0}\right)$ where $A_{x}: \mathbb{R}^{n} \rightarrow T_{x} M$ is a linear isomorphism and 
$A_{x}^{*}$ is the associated map $\operatorname{Sym}^{+}\left(\mathbb{R}^{n}\right) \rightarrow \operatorname{Sym}^{+}\left(T_{x} M\right)$. If the dependence $x \rightarrow A_{x}$ is smooth, then $\mathcal{C}$ is a GCS, which as in the standard conformal case, gives rise to a fibration $\mathcal{C} \rightarrow M$.

Let us here mention one useful and beautiful property of this moduli space $\operatorname{Sym}^{+}\left(\mathbb{R}^{n}\right)$, or more generally any $\operatorname{Sym}^{+}(E)$, for $E$ a linear space; this is the space of "linear" Riemannian metrics on $E$, and it admits itself a canonical Riemannian metric, which makes it as a universal symmetric space under the natural action of $\mathrm{GL}(E)$ (see $\S 3.2 .1$ ).

Let $H$ be the stabilizer subgroup in $\mathrm{GL}(n, \mathbb{R})$ of $\mathcal{C}_{0}$. For any $x \in M$, consider $I_{x}$ the set of isomorphisms $T_{x} M \rightarrow \mathbb{R}^{n}$ sending $\mathcal{C}_{x}$ to $\mathcal{C}_{0}$. This is clearly an $H$-orbit in the $\mathrm{GL}(n, \mathbb{R})$-space $\operatorname{Isom}\left(T_{x} M, \mathbb{R}^{n}\right)$, that is the fiber over $x$ of the frame bundle $P_{M} \rightarrow M$. When $x$ runs over $M$, we therefore get a section of $P_{M} / H \rightarrow M$, that is an $H$-structure on $M$.

Conversely, an $H$-structure gives naturally a GCS of type $\mathcal{C}_{0}$. Indeed, by definition of an $H$-structure, it consists in giving for any $x$, an $H$-orbit $I_{x}$ as above. The pull back $\mathcal{C}_{x}$ of the curve $\mathcal{C}_{0}$ by any element of $I_{x}$ does not depend on the choice of such element.

1.0.4. Rigidity. Let $\phi$ be a diffeomorphism of $M$ and $\phi^{*}$ its induced action on $\operatorname{Sym}(T M)$. Then $\phi$ is an automorphism of $\mathcal{C}($ a GCS on $M)$ if $\phi^{*}(\mathcal{C})=\mathcal{C}$.

The following discussion applies to diffeomorphisms sending a point $p \in M$ to another $q \in M$, but we will be specially interested in the case $p=q$. Then, define $\phi$ to be isometric up to order 1 at $p$, if $\phi(p)=p$ and $\left(\phi^{*}(\mathcal{C})\right)_{p}=\mathcal{C}_{p}$, i.e. $\phi^{*}(\mathcal{C})$ and $\mathcal{C}$ meet along $\mathcal{C}_{p}$. We say that $\phi$ is isometric up to order $d \geq 1$ at $p$ or simply a $d$-isometry if $\phi^{*}(\mathcal{C})$ and $\mathcal{C}$ have contact of order $(d-1)$ along $\mathcal{C}_{p}$. In order to be complete, let us precise that the local model of two $k$-submanifolds $V$ and $W$ of $\mathbb{R}^{N}$ having a contact at order $s$ along a curve $\mathcal{C}_{0}$, is that where $V=\mathbb{R}^{k}, \mathcal{C}_{0}=\mathbb{R} \subset \mathbb{R}^{k}$, and $W$ is the graph of a function $f: \mathbb{R}^{k} \rightarrow \mathbb{R}^{N-k}$ having a vanishing Taylor expansion up to order $s$ at all points of $\mathcal{C}_{0}$. Let us also indicate that we will say that $\phi$ has a trivial $d$-jet at $p$ if it has the same $d$-jet as the identity at $p$.

Rigidity at order 2 of classical conformal structures in dimension $\geq 3$, is essentially equivalent to the classical Liouville Theorem stating that any local conformal transformation of a Euclidean space of dimension $\geq 3$, is a composition of a translation, a similarity and an inversion (see for instance [6, 15] and [10]). There are many approaches to this rigidity, including that by the theory of $H$-structures of finite type, via computation of the prolongation spaces for the conformal group $H=\mathbb{R} . \mathrm{O}(n)$, see $[14,16,12,1]$. Here we generalize to generic GCS:

Theorem 1.1 (Generalized Liouville Theorem). Let $\mathcal{C}$ be a generic generalized conformal structure on a manifold of dimension $\geq 3$. Then $\mathcal{C}$ is d-rigid, for any $d \geq 2$, that is a $(d+1)$-isometry at a given point with a trivial $d$-jet, has a trivial $(d+1)$-jet. 
An alternative formulation would be that if a $(d+1)$-isometry at some point $p$, with $d \geq 2$, has a trivial 2 -jet then it has a trivial $(d+1)$-jet. In particular, if a smooth local isometry has a trivial 2 -jet at $p$, then it has trivial infinite jet, i.e. it is infinitely tangent to the identity at $p$.

In a first version of the present article we just proved 2-rigidity, we then investigate the general case after request of the referee. In fact, in the case of geometric structures in the Gromov sense, it is a general fact that $k$-rigidity implies $d$-rigidity for any $d \geq k$, and that a local isometry with a trivial $k$-jet at some point is the identity in a neighbourhood of it. These implications are somehow "tautological" but follow from a highly sophisticated machinery. Adaptation of this formalism to our situation seems possible but needs a specific and independent investigation, see $\S 2.2 .3$ and $\S 2.2 .4$ for a preliminary discussion on these aspects. Actually, our proof of $d$-rigidity of GCS for $d>2$ is done by rather adapting computations of the case $d=2$.

One essential motivation behind the Gromov notion of $k$-rigidity for a geometric structure is that it gives a way to prove that its isometry group is of Lie type. We will give details about this question of Lie group structure of isometry groups of GCS (as well as lightlike metrics) in a forthcoming article [4].

Example 1.2 (A non rigid example). Consider canonical coordinates $\left(x^{1}, \ldots, x^{n}\right)$ on $\mathbb{R}^{n}$. Endow it with $\mathcal{C}$ the "constant" GCS given by the curve of Euclidean metrics $t\left(d x^{1}\right)^{2}+\left(d x^{2}\right)^{2}+\ldots+\left(d x^{n}\right)^{2}, t>0$. This $\mathcal{C}$ is in fact an $H$-structure. Any diffeomorphism $\phi$ of the form $\phi\left(x^{1}, \ldots, x^{n}\right)=\left(f\left(x^{1}\right), x^{2}, \ldots, x^{n}\right)$ is isometric. This structure is not rigid, indeed $\mathcal{C}$ is not generic.

Note however that it may happen for a GCS to be rigid, even if it is not generic (such a situation is thus not covered by our result). For instance, for an $H$-structure with $H$ a one parameter subgroup of $\mathrm{GL}(n, \mathbb{R})$, one can prove it has finite type iff the Lie subalgebra of $H$ contains no matrices of rank 1, in which case the structure has finite type 1, i.e. it is 1-rigid like a Riemannian metric (see for instance ([14], page 4) for one implication).

Remark 1.3. More generalizations of conformal structures can be obtained by relaxing the dimension condition on $\mathcal{C}$, say by assuming $\operatorname{dim} \mathcal{C}=\operatorname{dim} M+l$, where $l$ may be bigger than 1 . The rigidity discussion will then depend on $l$ and $\operatorname{dim} M$ ?

\section{Further investigations}

2.1. Interplay with Lightlike metrics. Our motivation behind the study of GC structures was in fact their relation with the lightlike ones that we considered in [3]. Recall that a lightlike metric $g$ on a manifold $\mathcal{V}$ is a tensor which is a positive non-definite quadratic form of 1-dimensional kernel in each tangent space of $\mathcal{V}$ [2]. The kernel of $g$ is a direction field $N$, tangent to a 1-dimensional foliation $\mathcal{N}$ called null or characteristic. 
This null foliation is not necessarily oriented by a (global) non-singular vector field $X$ tangent to it, but we can assume it is the case by passing to a double cover, or arguing locally. Then, the lightlike structure is said to be transversally Riemannian if the Lie derivative $L_{X} g=0$. Let us say $g$ is nowhere transversally Riemannian, if $L_{X} g(x) \neq 0$, for any $x$. In the stronger situation where $L_{X} g$ is non-degenerate on $T \mathcal{V} / N, g$ is said to be generic. Both this genericity condition or being transversally Riemannian are independent of the choice of a particular $X$ orienting $\mathcal{N}$.

2.1.1. From GCS to lightlike structures. Let $\mathcal{C} \subset \mathrm{Sym}^{+}(T M)$ be a GCS on $M$ and $\pi: \mathcal{C} \rightarrow M$ the projection. Let $x \in M, q \in \mathcal{C}_{x}=\pi^{-1}(x)$, and consider the projection $d_{q} \pi: T_{q} \mathcal{C} \rightarrow T_{x} M$. Now, let $q$ play the role of a (definite) scalar product on $T_{x} M$, its pull back by $d_{q} \pi$ is a lightlike scalar product on $T_{q} \mathcal{C}$. We get in this way a tautological lightlike metric on $\mathcal{C}$.

Observe that this lightlike metric on $\mathcal{C}$ is nowhere transversally Riemannian, and also that $\mathcal{C}$ is generic as a GCS iff its lightlike metric is generic (as defined previously). To see all this, one writes all things in a local chart. If $x=\left(x^{1}, \ldots, x^{n}\right)$ are local coordinates on $M$, then $\mathcal{C}$ admits a parameterization $(t, x) \rightarrow c(t, x) \in$ $\operatorname{Sym}^{+}\left(T \mathbb{R}^{n}\right)$ (one can take $c$ of the form $\left.c(t, x)=(d(t, x), x) \in \operatorname{Sym}^{+}\left(\mathbb{R}^{n}\right) \times \mathbb{R}^{n}\right)$. The lightlike metric is defined by $g\left(\frac{\partial c}{\partial x^{i}}, \frac{\partial c}{\partial x^{j}}\right)=c(t, x)\left(\frac{\partial}{\partial x^{i}}, \frac{\partial}{\partial x^{j}}\right)$ (this last expression just means application of the scalar product $d(t, x)$ to $\left.\left(\frac{\partial}{\partial x^{2}}, \frac{\partial}{\partial x^{j}}\right)\right)$. If one takes $X=\frac{\partial}{\partial t}$ as a vector field tangent to the null direction, then $L_{X} g\left(\frac{\partial c}{\partial x^{2}}, \frac{\partial c}{\partial x^{j}}\right)=\frac{\partial c}{\partial t}\left(\frac{\partial}{\partial x^{2}}, \frac{\partial}{\partial x^{j}}\right)$. Now, for a given $x, t \rightarrow c(t, x)$ is a parameterization of $\mathcal{C}_{x}$ which is by our definition of a regular GCS, an embedded 1-dimensional manifold. Hence $\frac{\partial c}{\partial t}$ (seen as element of $\operatorname{Sym}\left(\mathbb{R}^{n}\right)$ ) does not vanish which shows that the associated lightlike structure is always nowhere transversally Riemannian. The lightlike metric $g$ is generic iff $L_{X} g$ is non-degenerate on the space generated by the $\frac{\partial c}{\partial x^{\imath}}$ 's. This is equivalent to that $\frac{\partial c}{\partial t}$ is non-degenerate, that is $\mathcal{C}$ is generic.

2.1.2. From lightlike to GCS structures. We will introduce a notion of simple lightlike manifold ensuring that it comes from a GCS.

Definition 1. A lightlike manifold $(\mathcal{V}, g)$ is said to be simple if

a) There is a Hausdorff manifold $M$ and a submersion $\pi: \mathcal{V} \rightarrow M$, such that the connected components of its levels are the leaves of the null foliation $\mathcal{N}$.

b) $\mathcal{C}$ is a regular $G C S$ on $M$, where for $x \in M, \mathcal{C}_{x}$ is the set of all scalar products obtained from the projections $T_{y} \mathcal{V} \rightarrow T_{x} M$, where $y \in \mathcal{V}$ is such that $x=\pi(y)$.

It is not so easy to formulate directly condition (b) by means of $(\mathcal{V}, g)$ only (without refereeing to $M$ ), but the condition implies in particular that $(\mathcal{V}, g)$ is nowhere transversally Riemannian. Conversely, and this is the point, a nowhere transversally Riemannian lightlike manifold is locally simple: any point admits a simple neighborhood. 
Summarizing: there is a one to one correspondence between GCS structures and simple lightlike ones, the generic in one hand correspond to the generic in the other, and locally any nowhere transversally Riemannian lightlike metric gives rise to a GCS.

Example 2.1. For the classical conformal sphere $\mathbb{S}^{n}$, the associated lightlike manifold $\mathcal{V}$ is the Minkowski lightcone

$$
\mathrm{Co}^{n+1}=\left\{x=\left(x^{1}, \ldots, x^{n+2}\right) \in \mathbb{R}^{n+2} / q(x)=0, x^{n+2}>0\right\}
$$

seen as a lightlike submanifold in the Minkowski space $\left(\mathbb{R}^{n+2}, q\right)$, where $q(x)=$ $\left(x^{1}\right)^{2}+\ldots+\left(x^{n+1}\right)^{2}-\left(x^{n+2}\right)^{2}$.

2.1.3. Sub-rigidity. A lightlike structure is an $H$-structure for $H$ the orthogonal group of the standard lightlike scalar product $\left(x^{1}\right)^{2}+\ldots+\left(x^{n-1}\right)^{2}$ on $\mathbb{R}^{n}$. This structure has infinite type in Cartan's terminology, equivalently it is not rigid in Gromov sense. We discussed in [3] subrigidity, a weaker property, that may be satisfied by lightlike metrics. For $i<d$, a geometric structure is $(d, i)$ subrigid, if any $d$-isometry which has a trivial $i$-jet at some point has in fact a trivial $(i+1)$-jet at that point. In particular, $(d+1, d)$ subrigidity coincides with usual $d$-rigidity.

2.1.4. Isometry groups. Let us call a transvection of $(\mathcal{V}, g)$ any map $\mathcal{V} \rightarrow \mathcal{V}$ sending each leaf of $\mathcal{N}$ to itself. A transvection is not necessarily isometric. In fact, any point admits in its neighborhood a non-singular vector field generating (local) transvections, iff $(\mathcal{V}, g)$ is transversally Riemannian.

If $(\mathcal{V}, g)$ is simple, then we have a group morphism $\operatorname{Iso}(\mathcal{V}, g) \rightarrow \operatorname{Iso}(M, \mathcal{C})$. Its kernel is $\operatorname{Iso}^{\operatorname{Tr}}(\mathcal{V}, g)$, the group of isometric transvections. In the simple case, Iso ${ }^{\operatorname{Tr}}(\mathcal{V}, g)$ does not contain one parameter groups, but we can not conclude it is discrete, for instance because one does not know if $\operatorname{Iso}(\mathcal{V}, g)$ is a Lie group.

Now, comparison between infinitesimal isometry groups of $(\mathcal{V}, g)$ and $(M, \mathcal{C})$ is even more complicated. We can however, as stated in [3], relate subrigidity of $(\mathcal{V}, g)$ to the rigidity of $(M, \mathcal{C})$. Our second main result in the present article will be to provide a proof of $(d+2, d)$ subrigidity of lightlike metrics based on Liouville Theorem for GCS:

Theorem 2.2. In dimension $\geq 4$, a generic lightlike metric is $(d+2, d)$ subrigid for $d \geq 1$, that is a (d+2)-isometry at a given point with a trivial d-jet has a trivial $(d+1)$-jet. In particular, an isometry with a trivial 1-jet at some point has a trivial infinite jet.

The proof will be given in $\S 6$. The general case is no more difficult than that of $d=1$, that is $(3,1)$-subrigidity. We will start giving a detailed proof in this last case and show afterwards adaptations to the higher order case $d>1$. 
2.2. Remarks on other aspects. Many other natural questions can be asked about both local and global properties of GCS. For instance, one may try to weaken the genericity condition in Theorems 1.1 and 2.2, and also study global properties of isometric actions preserving GCS from the point of view of a global rigidity, say by asking a conjecture of Lichnerowicz type (see [12, 8, 11]). We will here briefly discuss the following other aspects:

2.2.1. Pseudo-Riemannian case. If one replaces $\mathrm{Sym}^{+}$by Sym*, that is the space of non-degenerate quadratic forms (i.e. scalar pseudo-products) then one gets pseudoRiemannian GCS that are defined similarly by giving a curve in each $\operatorname{Sym}^{*}\left(T_{x} M\right)$, for $x \in M$. Theorem 1.1 seems to extend to this wider framework. Indeed, all algebraic and local computations in Sections 4 and 5 apply in this situation, since they do not assume positiveness but rather non-degeneracy of metrics. However, for the proof of Theorem 1.1, positiveness is required in particular to treat the periodic case 3.2 .

2.2.2. Anosov flows. Let us give hints that Anosov flows always preserve GCS (of Riemannian type), although they never preserve classical Riemannian conformal structures (see for instance [13] for basic notions). Indeed, this will be a particular case of the general construction of 1.0.1. The point is that, one can choose the initial Riemannian metric $g_{0}$ so that the corresponding family $\phi_{*}^{t} g_{0}$ defines a regular GCS. Essentially, for any $x, t \rightarrow\left(\phi_{*}^{t} g_{0}\right)(x) \in \mathrm{Sym}^{+}\left(T_{x} M\right)$ is a properly embedded curve $\mathcal{C}_{x}$, and thus $\mathcal{C}=\cup_{x} \mathcal{C}_{x}$ is a submanifold in $\operatorname{Sym}^{+}(T M)$. To ensure this, one has to start with an adapted $g_{0}$, that is, it is contracted on the stable bundle, and expanded on the unstable one.

Regarding genericity, let us make the following technical assumption (which it seems that one can overcome). Denote by $X$ the generating vector field of $\phi^{t}$. Then assume that $\phi^{t}$ preserves a smooth supplementary sub-bundle $E \subset T M$, i.e. $T M=\mathbb{R} X \oplus E$ (such an $E$ must be the sum of the stable and unstable bundles). Say $E$ is defined by a 1 differential form $\eta$. Assume $g_{0}(X, X)=1$, and consider now the GCS defined by $\phi_{*}^{t} g_{0}+f(t) \eta \otimes \eta$, with $f(t)$ and $\frac{\partial f}{\partial t}$ positive for any $t$. This GCS is generic.

2.2.3. A Geometric structure? In general, GCS are neither $H$-structures in Cartan sense nor geometric structures in the Gromov sense (see $[12,8,1,7])$ ! We already saw that a GCS $\mathcal{C}$ is an $H$-structure iff it is infinitesimally homogeneous: all the curves $\mathcal{C}_{x} \subset \operatorname{Sym}^{+}\left(T_{x} M\right)$ are linearly equivalent to a same curve $\mathcal{C}_{0} \subset \operatorname{Sym}^{+}\left(\mathbb{R}^{n}\right)$, when $x \in M(\S 1.0 .3)$.

Now, more generally, one may ask in which situations $\mathcal{C}$ can be naturally seen as a geometric structure in the Gromov sense? We will not investigate this question in the present article since it hides many technical difficulties. Let us just say that roughly speaking, and at a formal level, one considers $\mathcal{X}$, the space of nonparameterized curves $\mathbb{R} \rightarrow \operatorname{Sym}^{+}\left(\mathbb{R}^{n}\right)$ of a given regularity $C^{k}$, that is the quotient 
space of $C^{k}\left(\mathbb{R}, \operatorname{Sym}^{+}\left(\mathbb{R}^{n}\right)\right.$ by the $\operatorname{Diff}^{k}(\mathbb{R})$-right composition action. Let $\mathcal{X}^{*}$ be the subspace of those curves whose image is an embedded 1-submanifold in $\operatorname{Sym}^{+}\left(\mathbb{R}^{n}\right)$. The group $\mathrm{GL}(n, \mathbb{R})$ acts on both $\mathcal{X}$ and $\mathcal{X}^{*}$. Let us restrict ourselves to the case of structures $\mathcal{C} \rightarrow M$ that are trivial topological fibrations with fiber $\mathbb{R}$ and where $M$ is an open subset of $\mathbb{R}^{n}$. Such a $\mathcal{C}$ is equivalent to giving a map $\sigma: M \rightarrow \mathcal{X}^{*}$. Roughly, one may think of $\mathcal{C}$ as a geometric structure in the Gromov sense, if the image of $\sigma$ is contained in a $\mathrm{GL}(n, \mathbb{R})$-invariant subset $\Sigma \subset \mathcal{X}^{*}$, which is a finite dimensional manifold. It is not clear how to formulate a general statement about a situation where such a $\Sigma$ exists. Let us however notice the following simple example. Consider $d$ an integer, and let $\Sigma^{\prime}$ be the set of elements of $\mathcal{X}$ given by polynomial maps $\mathbb{R} \rightarrow \operatorname{Sym}\left(\mathbb{R}^{n}\right)$ of degree $\leq d$, and take $\Sigma=\Sigma^{\prime} \cap \mathcal{X}^{*}$.

2.2.4. A-type? Observe now that in order to get a geometric structure of algebraic type (A-type), as defined in [12] (see also [8, 1]), one needs $\Sigma$ to be an algebraic manifold and the $\mathrm{GL}(n, \mathbb{R})$-action on it algebraic (see [1]).

But, rigid geometric structures of algebraic type satisfy the Gromov's open dense orbit Theorem, that is if the isometry pseudo-group of the structure has a dense orbit, then this one is open! In other words an open dense subset is locally homogeneous (see $[12,8,5,17]$ ). However, one can see in the previous Anosov case that there are examples where such a local homogeneous subset can not exist. We then conclude that there is no way to see such a GCS as a geometric structure of algebraic type!

\section{SOME PRELIMINARIES}

3.1. Case of $H$-structures. Let $H \subset \mathrm{GL}(n, \mathbb{R})$ be a closed subgroup and $\mathfrak{h} \subset$ $\operatorname{End}\left(\mathbb{R}^{n}\right)$ its Lie algebra. Recall that the space $\mathfrak{h}_{d}$ of $d$-prolongations is that of symmetric $(d+1)$-multi-linear maps $A: \mathbb{R}^{n} \times \ldots \times \mathbb{R}^{n} \rightarrow \mathbb{R}^{n}$, such that for any given $\left(u_{1}, \ldots, u_{d}\right)$, the endomorphism $u \rightarrow A\left(u, u_{1}, \ldots, u_{d}\right)$ belongs to $\mathfrak{h}$. If for some $d \geq 1, \mathfrak{h}_{d}=0$, one says that $H$ has finite type, with order the smallest such $d$.

\subsubsection{Algebraic structure.}

Lemma 3.1. Let $\mathcal{C}_{0}$ be a connected curve in $\operatorname{Sym}^{+}\left(\mathbb{R}^{n}\right)$ and $H$ the connected component of its stabilizer in $\mathrm{GL}(n, \mathbb{R})$. Then $H$ is semi-direct product $P \ltimes K$, where $K$ is compact and acts trivially on $\mathcal{C}_{0}$, and $P$ is either trivial or a one parameter group acting transitively on $\mathcal{C}_{0}$.

Proof. $\mathcal{C}_{0}$ inherits from $\operatorname{Sym}^{+}\left(\mathbb{R}^{n}\right)$ a Riemannian metric (see 3.2.1), and so by taking its parametrization by arc length, it becomes isometric to an open interval of $\mathbb{R}$. In the case where $\mathcal{C}_{0}$ is a proper interval, its length is finite and hence it has limit endpoints, which are fixed by $H$, and thus $H$ is compact in this case. Let us now consider the case where $\mathcal{C}_{0}$ is isometric to $\mathbb{R}$. 
We have a representation $\rho: H \rightarrow \mathrm{Iso}(\mathbb{R})$. The kernel $K$ of $\rho$ is compact since it is a closed subgroup in the orthogonal group $O(b)$, for any $b \in \mathcal{C}_{0}$.

Since $H$ is connected, $\rho(H)$ is either trivial or coincides with the translation group of $\mathbb{R}$. It then follows that if $H$ is not compact, then $H / K \sim \mathbb{R}$. In this case, let $P$ be any one parameter group that projects onto $\mathbb{R}$ (to see it exists take the one parameter group generated by any vector not in the Lie subalgebra of $K$ ). Thus $H$ is a semi-direct product $P \ltimes K$.

3.1.2. Finiteness of type. Write $P=\exp t R$, and let $\langle$,$\rangle be a scalar product pre-$ served by $K$ (as in the lemma above). An element of the Lie algebra $\mathfrak{h}$ of $H$ has the form $C+\alpha R$, where $C$ is antisymmetric $\left(C=-C^{*}\right)$. A 2-prolongation $A: \mathbb{R}^{n} \times \mathbb{R}^{n} \times \mathbb{R}^{n} \rightarrow \mathbb{R}^{n}$ of $\mathfrak{h}$ is symmetric and satisfies that $W \rightarrow A(U, V, W)$ belongs to $\mathfrak{h}$ for any $U, V$. Therefore $A$ satisfies a relation

$$
\left\langle A(U, V, W), W^{\prime}\right\rangle+\left\langle A\left(U, V, W^{\prime}\right), W\right\rangle=K(U, V)\left\langle\left(R+R^{*}\right) W, W^{\prime}\right\rangle
$$

for some $K$. As it will be seen later on, this is exactly the equation (1) in the generalized Braid Lemma 4.2 with $J=\langle$,$\rangle and J^{\prime}(.,)=.\left\langle\left(R+R^{*}\right) .,.\right\rangle$. By this lemma, it follows that $\mathfrak{h}$ has type $\leq 2$ if the form $J^{\prime}$ is non-degenerate.

\subsection{Case of periodic curves.}

3.2.1. Metric on $\mathrm{Sym}^{+}$. Let $E$ be a vector space of dimension $n$. Its space of Euclidean Riemannian metrics (i.e. scalar products) $\mathrm{Sym}^{+}(E)$ admits itself a canonical Riemannian (but no longer Euclidean) metric. To see it, observe first that $\mathrm{Sym}^{+}(E)$ is an open set in $\operatorname{Sym}(E)$, and hence the tangent space $T_{b}\left(\operatorname{Sym}^{+}(E)\right)$ at any point $b$ can be identified with $\operatorname{Sym}(E)$. But a scalar product $b$ defines a scalar product $\bar{b}$ on $\operatorname{Sym}(E)$ : if $\left(e_{i}\right)$ is a $b$-orthonormal basis, then $e_{i}^{*} \otimes e_{j}^{*}$ is a $\bar{b}$-orthonormal basis, where $\left(e_{i}^{*}\right)$ is the dual basis (one has to check this does not depend on the basis). Now, endow $T_{b} \operatorname{Sym}^{+}(E)$ with $\bar{b}$. Clearly, if $F$ is another vector space, then any isomorphism $E \rightarrow F$ induces an isometry $\operatorname{Sym}^{+}(E) \rightarrow \operatorname{Sym}^{+}(F)$. In fact, $\operatorname{Sym}^{+}(E)$ is a symmetric space $\mathrm{GL}(E) / \mathrm{O}(b)$, where $\mathrm{O}(b)$ is the orthogonal group of any $b \in \operatorname{Sym}^{+}(E)$.

As an example for $E=\mathbb{R}$, one gets the metric $\frac{d x^{2}}{x^{2}}$ on $\mathbb{R}^{*}$, and for $E=\mathbb{R}^{2}$, one gets the direct product $\mathbb{H}^{2} \times \mathbb{R}$ (where $\mathbb{H}^{2}$ is the hyperbolic plane).

\subsubsection{Topology.}

Lemma 3.2. Let $\mathcal{C}$ be a $G C S$ on $M$ and assume that for some $x_{0} \in M, \mathcal{C}_{x_{0}}$ is a circle, i.e. a connected compact 1-manifold. Then, the same is true for nearby points. More precisely, there is a neighborhood $V$ of $\mathcal{C}_{x_{0}}$ in $\mathcal{C}$ and $U$ a neighborhood of $x_{0}$ such that $\pi: V \rightarrow U$ is a Seifert fibration.

Proof. Let $I$ be a small arc in $M$ containing $x_{0}$, then $S=\pi^{-1}(I)$ is a surface containing $\mathcal{C}_{x_{0}}$. Let $S_{0}$ be the connected component of $\mathcal{C}_{x_{0}}$ in $S$. For $I$ small 
enough, $S_{0}$ is a tubular neighborhood of $\mathcal{C}_{x_{0}}$ in $S$, and it is thus an annulus or a Moebius strip around $\mathcal{C}_{x_{0}}$.

Let us start considering the annulus case. When $x$ runs over $I$, the connected components of the $\mathcal{C}_{x}$ in $S_{0}$ determine a 1-dimensional foliation $\mathcal{F}$ of $S_{0}$. But, each $\mathcal{C}_{x}$ is closed in $\mathcal{C}$ and hence each $\mathcal{F}$-leaf is closed. But such a foliation on the annulus is trivial, i.e. a trivial fibration on the interval

Now, consider the same foliation $\mathcal{F}$, but on a neighborhood $V=\pi^{-1}(U)$ in $\mathcal{C}$, where $U$ is a small neighborhood of $x_{0}$ in $M$. Since $U$ can be generated by arcs, leaves of $\mathcal{F}$ are all closed. But the holonomy of $\mathcal{C}_{x_{0}}$ in $U$ is trivial, since it is so above any interval. Hence the foliation is a fibration.

Consider now the case where for some $\operatorname{arcs} I, \pi^{-1}(I)$ is a Moebius strip. Then, on such a surface, the foliation $\mathcal{F}$ is a Seifert fibration with monodromy $\mathbb{Z} / 2 \mathbb{Z}$. As above, generate a neighborhood $U$ by arcs such that the holonomy on each of them is either trivial or has order 2. It follows that the (global) holonomy has order 2, and hence $\mathcal{F}$ is given by a Seifert fibration.

\subsubsection{Geometry.}

Proposition 3.3. If $\mathcal{C}$ has a circle fiber $\mathcal{C}_{x_{0}}$, then it is 1-rigid at $x_{0}$. In fact, $\mathcal{C}$ determines naturally a Riemannian metric near $x_{0}$.

Proof. For all $x, \mathcal{C}_{x}$ is a circle in $\operatorname{Sym}^{+}\left(T_{x} M\right)$. Consider an arc length parameterization $t \in[0, l] \rightarrow f(t) \in \mathcal{C}_{x}$, where $l$ is the length of $\mathcal{C}_{x}$ ( $f$ is defined up to a choice of an origin). The mean $\int f(t) d t$ is a canonically defined element of $\mathrm{Sym}^{+}\left(T_{x} M\right)$, call it $g_{x}$. Since $\pi$ is a smooth fibration, $g_{x}$ depends smoothly on $x$, that is $g$ is a smooth Riemannian metric defined on a neighborhood of $x_{0}$.

One then verifies that a $d$-isometry of $\mathcal{C}$ is a $d$-isometry for $g$. In order to check it, one considers the mapping which associates $g$ to $\mathcal{C}$, say $F: \mathcal{G} \rightarrow \mathcal{M}$, defined on the space of GCS with circle fibers, and having as a target the space of Riemannian metrics $\mathcal{M}$. Locally, an element of $\mathcal{G}$ is a mapping $M \rightarrow \mathcal{L}$, where $\mathcal{L}$ is the space of arc-length parameterized circle maps $\mathbb{S}^{1} \rightarrow \operatorname{Sym}\left(\mathbb{R}^{n}\right)$. The mapping $F: \mathcal{G} \rightarrow \mathcal{M}$ is just a mean, and therefore smooth. From all these constructions follows that if $\mathcal{C}$ and $\mathcal{C}^{\prime}$ have contact up to order $d$ at $p$, then the same is true for $g=F(\mathcal{C})$ and $g^{\prime}=F\left(\mathcal{C}^{\prime}\right)$. Applying this to $\mathcal{C}^{\prime}=\phi^{*}(\mathcal{C})$ yields that a $d$-isometry for $\mathcal{C}($ at $p$ ) is a $d$-isometry for $g$.

Finally, by 1-rigidity of Riemannian metrics (that is a 2-isometry with trivial 1 -jet has a trivial 2 -jet) we deduce that $\mathcal{C}$ is 1 -rigid.

\section{A generalized Braid Lemma}

The classical well known Braid Lemma (see for instance [6]) states: 
Lemma 4.1. [Braid Lemma] If $L$ is a trilinear map $E \times E \times E \rightarrow E$ on a vector space $E$, such that $L$ is symmetric on the two first variables and skew-symmetric on the two last ones, then $L=0$. In particular, if $A$ is a bilinear map $E \times E \rightarrow E$ such that

$$
<A(U, V), W>+<A(U, W), V>=0 \text { for all } U, V \text { and } W \text { in } E,
$$

where $\langle$,$\rangle is a Euclidean scalar product, then A=0$.

If fact this is also true for pseudo-scalar products, that is for $\langle$,$\rangle replaced by any$ non-degenerate symmetric bilinear form.

This statement is equivalent to the vanishing of 1-prolongations of the orthogonal group $O(E,\langle\rangle$,$) , and thus to the 1-rigidity of a Riemannian structures.$

We are going here to give a generalized Braid Lemma adapted to GC structures, which is in fact a slight generalization of the classical result on vanishing of second prolongations of $\mathfrak{c o}(n)$, see for instance [1] and ([16], page 335). Now, $A$ will be a trilinear symmetric map $E \times E \times E \rightarrow E$, where $E$ is a vector space which will be always assumed to have dimension $\geq 3$.

Proposition 4.2. [Generalized Braid Lemma] Let A be a symmetric 3-linear vectorial form $E \times E \times E \rightarrow E$ satisfying:

$$
J\left(A(U, V, W), W^{\prime}\right)+J\left(A\left(U, V, W^{\prime}\right), W\right)=K(U, V) J^{\prime}\left(W, W^{\prime}\right)
$$

where $J, J^{\prime}$ and $K: E \times E \rightarrow \mathbb{R}$ are some symmetric bilinear forms.

If $J$ and $J^{\prime}$ are non-degenerate, then $A=0$.

Proof. A direct computation gives us:

$$
\begin{aligned}
K(U, V) J^{\prime}\left(W, W^{\prime}\right) & +K\left(W, W^{\prime}\right) J^{\prime}(U, V) \\
& =K(U, W) J^{\prime}\left(V, W^{\prime}\right)+K\left(V, W^{\prime}\right) J^{\prime}(U, W)
\end{aligned}
$$

(One just replaces each term as $K(U, V) J^{\prime}\left(W, W^{\prime}\right)$ by its equivalent in the right hand of (1), and uses the fact that $A$ is symmetric).

Now let $W_{1}$ and $W_{2}$ be two $J^{\prime}$-orthogonal vectors: $J^{\prime}\left(W_{1}, W_{2}\right)=0$. Let $W_{3}$ be a third vector $J^{\prime}$-orthogonal to $\mathbb{R} W_{1}+\mathbb{R} W_{2}$ and $J^{\prime}\left(W_{3}, W_{3}\right) \neq 0$. Such $W_{3}$ exists because $\operatorname{dim} E \geq 3$ and $J^{\prime}$ is non-degenerate. We have:

$$
\begin{aligned}
K\left(W_{1}, W_{2}\right) J^{\prime}\left(W_{3}, W_{3}\right) & +K\left(W_{3}, W_{3}\right) J^{\prime}\left(W_{1}, W_{2}\right) \\
& =K\left(W_{1}, W_{3}\right) J^{\prime}\left(W_{2}, W_{3}\right)+K\left(W_{2}, W_{3}\right) J^{\prime}\left(W_{1}, W_{3}\right)
\end{aligned}
$$

which implies $K\left(W_{1}, W_{2}\right)=0$.

Write $K(U, V)=J^{\prime}(U, P(V))$, where $P$ is a $J^{\prime}$-symmetric endomorphism of $E$.

Let $W_{1}$ with $J^{\prime}\left(W_{1}, W_{1}\right) \neq 0$, and denote by $W_{1}^{\perp}$ its $J^{\prime}$-orthogonal. It follows that $P\left(W_{1}\right)$ is orthogonal to $W_{1}^{\perp}$, and hence $P\left(W_{1}\right) \in \mathbb{R} W_{1}$, that is $W_{1}$ is an eigenvector of $P$.

Thus $P$ has all vectors $W_{1}$ with non-vanishing $J^{\prime}\left(W_{1}, W_{1}\right)$ as eigenvectors. It follows that $P$ is a homothety, that is $K=\alpha J^{\prime}$ for some $\alpha \in \mathbb{R}$. 
Now, using (2) for $V=U, W^{\prime}=W$ and $J^{\prime}(U, W)=0$, we get:

$$
\alpha J^{\prime}(U, U) J^{\prime}(W, W)=0
$$

which implies $\alpha=0$ (since we can easily choose $U$ and $W$ with non-vanishing (square) $J^{\prime}$-norm). Therefore, (1) becomes

$$
J\left(A(U, V, W), W^{\prime}\right)+J\left(A\left(U, V, W^{\prime}\right), W\right)=0
$$

which implies by the classical Braid Lemma that $A=0$.

\section{Proof of the generalized Liouville Theorem}

5.1. Set-up of the problem. Let $(M, \mathcal{C})$ be a GC manifold. The investigations in the present section are local in nature, so the manifold $M$ can be identified with an open set in $\mathbb{R}^{n}$ with coordinates $\left(x^{1}, \ldots, x^{n}\right)$. In fact, we will work on a small neighborhood of a fixed point $p$.

So far, we have studied the situation where a component of $\mathcal{C}_{p}$ is a circle, and proved 1-rigidity in this case.

So we will now consider the opposite situation where all components of $\mathcal{C}_{p}$ are injective images of $\mathbb{R}$. We choose one component and analyze $\mathcal{C}$ around it. The projection $\pi$ is not necessarily a locally trivial fibration, but restricting to a small neighborhood of $p$ (that we will still denote $M$ ), any neighbourhood of a bounded arc of $\mathcal{C}_{p}$ can be parameterized by a map

$$
J: M \times I \rightarrow J(x, r) \in \operatorname{Sym}^{+}\left(T_{x} M\right)
$$

where $I$ is a bounded interval of $\mathbb{R}$. We can also assume that $r \rightarrow J(x, r)$ is an arc length parameterization, for any $x$, although we do not need it. So locally,

$$
J(x, r)=\sum_{i, j} a_{i j}(x, r) d x^{i} d x^{j}
$$

We will always assume that the associated lightlike structure is nowhere transversally Riemannian, that $\partial_{r} J \neq 0$.

5.1.1. Isometries. For $\phi$ a diffeomorphism of $M, \phi_{x}^{\prime}$ denotes its derivative at $x$.

A diffeomorphism $\phi$ is isometric if its natural action on $\operatorname{Sym}^{+}(T M)$ preserves $\mathcal{C}$, that is $\phi_{x}^{\prime}\left(\mathcal{C}_{x}\right)=\mathcal{C}_{\phi(x)}$. If the parameterization $J$ were global, then the isometric property implies the existence of a re-parameterization $(x, r) \rightarrow k(x, r) \in \mathbb{R}$, such that:

$$
J(\phi(x), k(x, r))\left(\left(\phi_{x}^{\prime}\right)(U),\left(\phi_{x}^{\prime}\right)(V)\right)-J(x, r)(U, V)=0, \forall U, V \text { vector fields. }
$$

Remark that, although we will not use it, if the $\mathcal{C}$-curves are parameterized by arc length, then $k$ has the form $k(x, r)=\delta(x)+r$.

Now, if the parameterization is not global, one just has to take care of the domains of definition; the same equation remains true. Actually, one has a map $(x, r) \in M_{1} \times I \rightarrow(\phi(x), k(x, r)) \in M_{2} \times K$, where $K$ is another interval, $M_{1}$ and 
$M_{2}$ are open subsets of $M$. However, for the sake of simplicity of notation, we will argue as if the parameterization is global, say $I=K$, and also $M_{1}=M_{2}=M$.

\subsection{Notation.}

The notation $\phi_{x}^{\prime}$ designs the total derivative of the diffeomorphism $\phi$. Second and third total derivatives are denoted $\phi_{x}^{\prime \prime}$ and $\phi_{x}^{\prime \prime \prime}$ respectively, the higher ones of order $m, m \in \mathbb{N}^{*}$, are denoted $\phi_{x}^{(m)}$. For a function $a$ on $(x, r)$ we denote the derivative with respect to $x$ at a point $(p, r)$ by $\mathbf{D}_{(p, r)} a$ (i.e. the differential of $x \rightarrow a(x, r)$ where $r$ is fixed). We similarly denote the same derivative of order $m$ by $\mathbf{D}_{(p, r)}^{(m)} a$. Regarding the derivative with respect to $r$ at a point $(p, r)$, we just denote it $\partial_{(p, r)} a$.

5.2.1. Infinitesimal isometries. Assume now that $\phi(p)=p$. By definition, $\phi$ is a $d$-isometry at $p$ if $\mathcal{C}$ and its image $\phi^{*}(\mathcal{C})$ have a contact at order $d$ along $\mathcal{C}_{p}$ (1.0.4). As in the classical case, one shows this is equivalent to the usual vanishing condition up to order $d$, at $p$, of the equality (3). More precisely, for a given function $k$, and $U, V$ vector fields, let

$$
\Delta_{k}(U, V)(x, r)=J(\phi(x), k(x, r))\left(\left(\phi_{x}^{\prime}\right)(U),\left(\phi_{x}^{\prime}\right)(V)\right)-J(x, r)(U, V)
$$

Then, $\phi$ is a $d$-isometry at $p$, if there exists a function $k$ such that the derivatives with respect to $x$ up to order $(d-1)$ of $\Delta_{k}(U, V)$ vanish at $(p, r)$, for any vector fields $U$ and $V$ and any $r$. Actually, it suffices to check this for $U$ and $V$ elements of a frame field on $M$, for example the natural vector fields $\frac{\partial}{\partial x^{i}}$. In the sequel, we will take $U$ and $V$ to be combination with constant coefficients of the $\frac{\partial}{\partial x^{i}}$.

5.3. $(d+1)$-Isometries for $d \geq 2$. If $\phi$ is an isometry of order $d+1$ at $p$, then for all $r$

$$
J(p, r)(U, V)=J(\phi(p), k(p, r))\left(\phi_{p}^{\prime}(U), \phi_{p}^{\prime}(V)\right), \forall U, V \in T_{p} M
$$

Taking derivative (with respect to $x$ ) at $p$ gives,

$$
\begin{aligned}
\mathbf{D}_{(p, r)} J\left(W_{1}\right)(U, V)= & \mathbf{D}_{(\phi(p), k(p, r))} J\left(\phi_{p}^{\prime}\left(W_{1}\right)\right)\left(\phi_{p}^{\prime}(U), \phi_{p}^{\prime}(V)\right) \\
& +\mathbf{D}_{(p, r)} k\left(W_{1}\right) \partial_{(\phi(p), k(p, r))} J\left(\phi_{p}^{\prime}(U), \phi_{p}^{\prime}(V)\right) \\
& +J(\phi(p), k(p, r))\left(\phi_{p}^{\prime \prime}\left(U, W_{1}\right), \phi_{p}^{\prime}(V)\right) \\
& +J(\phi(p), k(p, r))\left(\phi_{p}^{\prime}(U), \phi_{p}^{\prime \prime}\left(V, W_{1}\right)\right.
\end{aligned}
$$

for all $U, V, W_{1} \in T_{p} M$.

Lemma 5.1. Let $\phi$ be an isometry of order $(d+1)$ at $p$ with a d-trivial jet $\left(j^{j} t_{p}^{d}(\phi)=\right.$ 1) then, for all $r$

$$
k(p, r)=r \text { and } \mathbf{D}_{(p, r)}^{(m)} k=0 \text { for } 1 \leq m \leq d-1
$$

Proof. This will follow from taking derivatives of (4) up to order $d-1$. But, since $j e t_{p}^{d}(\phi)=1$, one can argue as if $\phi$ was the identity, that is (4) becomes

$$
J(p, r)(U, V)=J(p, k(p, r))(U, V), \forall U, V \in T_{p} M
$$


This formula involves $k$ only.

This equality itself implies that $k(p, r)=r$. Indeed, by our hypotheses (in the beginning of the present $\S), \mathcal{C}_{p}$ is a 1-dimensional submanifold without compact components, in particular, $r \rightarrow J(p, r)$ is injective, and hence $k(p, r)=r$.

To prove vanishing of $\mathbf{D}_{(p, r)} k$ (the differential of $k$ with respect to $x$ ), just differentiate the formula (6) (for instance by replacing in (5)) and get

$$
\mathbf{D}_{(p, r)} J\left(W_{1}\right)(U, V)=\mathbf{D}_{(p, r)} J\left(W_{1}\right)(U, V)+\mathbf{D}_{(p, r)} k\left(W_{1}\right) \partial_{(p, r)} J(U, V)
$$

which means

$$
\mathbf{D}_{(p, r)} k\left(W_{1}\right) \partial_{(p, r)} J(U, V)=0
$$

(Remember the notation $\partial_{(p, r)}$ introduced in $\left.\S 5.2\right)$. But, by definition of GCS, the curve $r \rightarrow J(p, r) \in \operatorname{Sym}^{+}\left(T_{p} M\right)$ is non-singular, and hence $(U, V) \rightarrow \partial_{(p, r)} J(U, V)$ is a non-vanishing bilinear form, and so $\mathbf{D}_{(p, r)} k=0$.

Finally, vanishing of higher order derivatives is done by induction. Assume $\mathbf{D}_{(p, r)}^{(m)} k=0$ for $m \leq l$ (with $1 \leq l \leq d-2$ ), and take the derivative of order $l+1$ of the equality (6) at $p$. All terms containing $\mathbf{D}_{(p, r)}^{(m)} k$ for $1 \leq m \leq l$ disappear and remains the equality

$$
\begin{aligned}
\mathbf{D}_{(p, r)}^{(l+1)} J\left(W_{1}, \ldots, W_{l+1}\right)(U, V)= & \mathbf{D}_{(p, r)}^{(l+1)} J\left(W_{1}, \ldots, W_{l+1}\right)(U, V) \\
& +\mathbf{D}_{(p, r)}^{(l+1)} k\left(W_{1}, \ldots, W_{l+1}\right) \partial_{(p, r)} J(U, V)
\end{aligned}
$$

for all $W_{1}, \ldots, W_{l+1}$ in $T_{p} M$, which implies (as in the case of $\mathbf{D}_{(p, r)} k$ )

$$
\mathbf{D}_{(p, r)}^{(l+1)} k=0
$$

Lemma 5.2. Let $\phi$ be an isometry of order $(d+1)$ at $p$ such that jet $t_{p}^{d}(\phi)=1$. Then, $\phi_{p}^{(d+1)}$ satisfies

$$
\begin{gathered}
J(p, r)\left(\phi_{p}^{(d+1)}\left(U, W_{1}, \ldots, W_{d}\right), V\right)+J(p, r)\left(U, \phi_{p}^{(d+1)}\left(V, W_{1}, \ldots, W_{d}\right)\right) \\
=-\mathbf{D}_{(p, r)}^{(d)} k\left(W_{1}, \ldots, W_{d}\right) \partial_{(p, r)} J(U, V)
\end{gathered}
$$

for any $r$ and all $W_{1}, \ldots, W_{d}, U, V$ in $T_{p} M$.

Proof. Computation of the derivative at order $(d-1)$ of $(5)$ at $p$, will be drastically simplified by the fact that $\phi_{p}^{\prime}=I d, \phi_{p}^{(m)}=0$ for $2 \leq m \leq d, k(p, r)=r$ and $\mathbf{D}_{(p, r)}^{(m)} k=0$ for $1 \leq m \leq d-1$ (by the previous lemma), and reduces exactly to

$$
\begin{gathered}
\mathbf{D}_{(p, r)}^{(d)} J\left(W_{1}, \ldots, W_{d}\right)(U, V)=\mathbf{D}_{(p, r)}^{(d)} J\left(W_{1}, \ldots, W_{d}\right)(U, V)+\mathbf{D}_{(p, r)}^{(d)} k\left(W_{1}, \ldots, W_{d}\right) \partial_{(p, r)} J(U, V) \\
+J(p, r)\left(\phi_{p}^{(d+1)}\left(U, W_{1}, \ldots, W_{d}\right), V\right)+J(p, r)\left(U, \phi_{p}^{(d+1)}\left(V, W_{1}, \ldots, W_{d}\right)\right)
\end{gathered}
$$

for all $W_{1}, \ldots, W_{d}, U, V$ in $T_{p} M$, witch is exactly (7). 
5.4. End of the proof of Theorem 1.1. With notations of the previous lemma, we have to prove that $\phi_{p}^{(d+1)}=0$. This will indeed follow form a straightforward application of the Generalized Braid Lemma 4.2 to (7).

For the sake of clarity, let us first start with the case $d=2$. So $\phi$ is a 3-isometry at $p$ with a trivial 2 -jet, thus by (7)

$$
\begin{gathered}
J(p, r)\left(\phi_{p}^{\prime \prime \prime}\left(U, W_{1}, W_{2}\right), V\right)+J(p, r)\left(U, \phi_{p}^{\prime \prime \prime}\left(V, W_{1}, W_{2}\right)\right. \\
=-\mathbf{D}_{(p, r)}^{(2)} k\left(W_{1}, W_{2}\right) \partial_{(p, r)} J(U, V)
\end{gathered}
$$

Apply the Generalized Braid Lemma with $A=\phi_{p}^{\prime \prime \prime}, J=J(p, r), K=\mathbf{D}_{(p, r)}^{2} k$ and $J^{\prime}=-\partial_{(p, r)} J$, which is actually non-degenerate by the genericity hypothesis on $\mathcal{C}$. Then conclude that $A=\phi_{p}^{\prime \prime \prime}=0$.

In the general case, $d>2$, apply the Generalized Braid Lemma with $J=J(p, r)$, $J^{\prime}=-\partial_{(p, r)} J$ and $K=\mathbf{D}_{(p, r)}^{(d)} k\left(., ., W_{3}, \ldots, W_{d}\right)$, where $W_{3}, \ldots, W_{d}$ are fixed vectors in $T_{p} M$. One gets that $A=\phi_{p}^{(d+1)}\left(., ., ., W_{3}, \ldots, W_{d}\right)=0$. But since $W_{3}, \ldots W_{d}$ are arbitrary, $\phi_{p}^{(d+1)}=0$.

\section{Sub-Rigidity of Lightlike metrics, Proof of Theorem 2.2}

6.1. Setting of the problem. Let $(\mathcal{V}, g)$ be a lightlike $n$-dimensional manifold. Since we are dealing with questions local in nature, so we can assume $\mathcal{V}$ is a small chart domain, say $\mathcal{V}=M \times I$ where $I$ is an interval. The factor $I$ corresponds to the characteristic foliation tangent to the kernel of $g$. In an adapted coordinate system $(x, t)=\left(x^{1}, x^{2}, \ldots, x^{n-1}, t\right)$ ( $t$ corresponds to $\left.I\right)$, the lightlike metric takes the form

$$
g_{(x, t)}=\sum_{i, j} a_{i j}(x, t) d x^{i} d x^{j}
$$

This gives for any fixed $r$, a Riemannian metric on $M \times\{r\}$. By endowing $T_{x} M$ with the scalar products $g_{(x, r)}, r \in I$, we get a GCS on $M$, once we assume $g$ nowhere transversally Riemannian, that is $\frac{\partial}{\partial t} g_{(x, t)} \neq 0$ (see 2.1). Recall that $g$ is said to be generic if $\frac{\partial}{\partial t} g_{(x, t)}=\sum_{i, j} \frac{\partial a_{i j}}{\partial t}(x, t) d x^{i} d x^{j}$ is non-degenerate.

A diffeomorphism $\Psi$ of $M$ has the form $\Psi=(\phi, \delta)$ where $\phi: M \times I \rightarrow M$ and $\delta: M \times I \rightarrow I$.

If $\Psi$ is isometric, then it preserves the $I$ foliation, and hence $\phi$ does not depend of $t$. Furthermore, for any $U$ and $V$ in $T_{(x, t)} \mathcal{V}$

$$
g_{(x, t)}(U, V)=g_{\Psi(x, t)}\left(\Psi_{(x, t)}^{\prime}(U), \Psi_{(x, t)}^{\prime}(V)\right)
$$

A tangent vector $U \in T_{(x, t)} \mathcal{V}$ will be denoted $\left(U_{M}, U_{I}\right) \in T_{x} M \times T_{t} I$.

As said after the statement of the Theorem, we will start giving the proof in the case $d=1$ which consists in three steps. The higher order case will be treated at $\S 6.5$. 
6.2. Step 1: a partial 1-rigidity. If $\Psi$ is isometric up to order 2 , with a trivial 1-jet at a point $(p, r) \in \mathcal{V}$ then $\phi_{(p, r)}^{\prime \prime}=0$.

Proof. If $\Psi=(\phi, \delta)$ is isometric up to order 2 then the equality of (8) holds for the derivatives at $(x, t)=(p, r)$. We have

$$
\left(g_{(x, t)}(U, V)\right)_{(p, r)}^{\prime}(W)=\sum_{i, j}\left(a_{i j}\right)_{(p, r)}^{\prime}(W)(U)_{i}(V)_{j}
$$

In the other hand, if we denote a generic point $(x, t)$ by $v$

$$
g_{\Psi(v)}\left(\Psi_{v}^{\prime}(U), \Psi_{v}^{\prime}(V)\right)=\sum_{i, j} a_{i j}(\Psi(v))\left(\Psi_{v}^{\prime}(U)\right)_{i}\left(\Psi_{v}^{\prime}(V)\right)_{j}
$$

a derivation gives

$$
\begin{gathered}
\sum_{i, j}\left(a_{i j}\right)_{\Psi(v)}^{\prime}\left(\Psi_{v}^{\prime}(W)\right)\left(\Psi_{v}^{\prime}(U)\right)_{i}\left(\Psi_{v}^{\prime}(V)\right)_{j}+\sum_{i, j} a_{i j}(\Psi(v))\left(\Psi_{v}^{\prime \prime}(U, W)\right)_{i}\left(\Psi_{v}^{\prime}(V)\right)_{j} \\
+\sum_{i, j} a_{i j}(\Psi(v))\left(\Psi_{v}^{\prime}(U)\right)_{i}\left(\Psi_{v}^{\prime \prime}(V, W)\right)_{j}
\end{gathered}
$$

We have

$$
\left(\Psi_{v}^{\prime}(U)\right)_{i}=\left(\phi_{v}^{\prime}(U)\right)_{i} \text { and }\left(\Psi_{v}^{\prime \prime}(U)\right)_{i}=\left(\phi_{v}^{\prime \prime}(U)\right)_{i}
$$

using the triviality of the 1 -jet of $\Psi$ we get

$$
\Psi(p, r)=(p, r), \Psi_{(p, r)}^{\prime}=I d
$$

then (10) becomes

$$
\sum_{i, j}\left(a_{i j}\right)_{(p, r)}^{\prime}(W)(U)_{i}(V)_{j}+\sum_{i, j} a_{i j}(p, r)\left(\phi_{(p, r)}^{\prime \prime}(U, W)\right)_{i}(V)_{j}+\sum_{i, j} a_{i j}(p, r)(U)_{i}\left(\phi_{(p, r)}^{\prime \prime}(V)\right)_{j}
$$

Therefore, the equality with (9) gives

$$
\sum_{i, j} a_{i j}(p, r)\left(\phi_{(p, r)}^{\prime \prime}(U, W)\right)_{i}(V)_{j}+\sum_{i, j} a_{i j}(p, r)(U)_{i}\left(\phi_{(p, r)}^{\prime \prime}(V, W)\right)_{j}=0
$$

that is

$$
g_{(p, r)}\left(\phi_{(p, r)}^{\prime \prime}(U, W), V\right)+g_{(p, r)}\left(U, \phi_{(p, r)}^{\prime \prime}(V, W)\right)=0
$$

By the Braid Lemma 4.1 we conclude that $\phi_{(p, r)}^{\prime \prime}=0$.

6.3. Step 2: the $\phi$-part. Assume the lightlike structure generic. If $\Psi=(\phi, \delta)$ is a 3-isometry at $(p, r)$ with a trivial 1-jet, then $\phi_{(p, r)}^{\prime \prime \prime}=0$.

Proof. If $\Psi$ was a true isometry, then it acts, via $\phi$, on $M$ seen as the quotient space of the characteristic foliation (in particular it does not depend on $r$ ), and it preserves the GCS on it. The genericity hypothesis allows one to apply Theorem 1.1 to conclude that $\phi_{(p, r)}^{\prime \prime \prime}=0$.

Now, we want to apply the same argument when $\Psi$ is merely isometric up to order 3 at $(p, r)$ (and has a trivial 1-jet). The idea then is to show that the diffeomorphism $x \rightarrow \varphi(x)=\phi(x, r)$ is a 3 -isometry of the GCS of $M$. The expected $k$-shift of $\varphi$ 
(see 5.1.1) will be nothing but $\delta$. In other words, we want $\varphi$ to satisfy the following equation up to order 3 at $p$ :

$$
g_{(x, r)}(U, V)=g_{(\varphi(x), \delta(x, r))}\left(\varphi_{x}^{\prime}(U), \varphi_{x}^{\prime}(V)\right)
$$

This property of $\varphi$, follows from the similar one of $\Phi$, that is, it satisfies (8) up to order 3 , and remembering that $\phi_{(p, r)}^{\prime \prime}=0$ by the previous step. Indeed, let us derive twice the equation satisfied by $\Psi$ at $\nu=(p, r)$ :

$$
g_{(x, t)}(U, V)=g_{\Psi(x, t)}\left(\Psi_{(x, t)}^{\prime}(U), \Psi_{(x, t)}^{\prime}(V)\right)
$$

we get, for all $W_{1}, W_{2} \in T_{(x, t} \mathcal{V}$,

$$
\begin{aligned}
g_{\nu}^{W_{1}, W_{2}}(U, V) & =g_{\Psi(\nu)}^{\Psi_{\nu}^{\prime \prime}\left(W_{1}, W_{2}\right)}\left(\Psi_{\nu}^{\prime}(U), \Psi_{\nu}^{\prime}(V)\right)+g_{\Psi(\nu)}^{\Psi_{\nu}^{\prime}\left(W_{1}\right), \Psi_{\nu}^{\prime}\left(W_{2}\right)}\left(\Psi_{\nu}^{\prime}(U), \Psi_{\nu}^{\prime}(V)\right) \\
& +g_{\Psi(\nu)}^{\Psi_{\nu}^{\prime}\left(W_{1}\right)}\left(\Psi_{\nu}^{\prime \prime}\left(U, W_{2}\right), \Psi_{\nu}^{\prime}(V)\right)+g_{\Psi(\nu)}^{\Psi_{\nu}^{\prime}\left(W_{1}\right)}\left(\Psi_{\nu}^{\prime}(U), \Psi_{\nu}^{\prime \prime}\left(V, W_{2}\right)\right) \\
& +g_{\Psi(\nu)}^{\Psi_{\nu}^{\prime}\left(W_{2}\right)}\left(\Psi_{\nu}^{\prime \prime}\left(U, W_{1}\right), \Psi_{\nu}^{\prime}(V)\right)+g_{\Psi(\nu)}^{\Psi_{\nu}^{\prime}\left(W_{2}\right)}\left(\Psi_{\nu}^{\prime}(U), \Psi_{\nu}^{\prime \prime}\left(V, W_{1}\right)\right) \\
& +g_{\Psi(\nu)}\left(\Psi_{\nu}^{\prime \prime}\left(U, W_{1}\right), \Psi_{\nu}^{\prime \prime}\left(V, W_{2}\right)\right)+g_{\Psi(\nu)}\left(\Psi_{\nu}^{\prime \prime}\left(U, W_{2}\right), \Psi_{\nu}^{\prime \prime}\left(V, W_{1}\right)\right) \\
& +g_{\Psi(\nu)}\left(\Psi_{\nu}^{\prime \prime \prime}\left(U, W_{1}, W_{2}\right), \Psi_{\nu}^{\prime}(V)\right)+g_{\Psi(\nu)}\left(\Psi_{\nu}^{\prime}(U), \Psi_{\nu}^{\prime \prime \prime}\left(V, W_{1}, W_{2}\right)\right)
\end{aligned}
$$

where

$$
g_{v}^{W}=\sum_{i, j}\left(a_{i j}\right)_{v}^{\prime}(W) d x^{i} d x^{j}
$$

and

$$
g_{v}^{W_{1}, W_{2}}=\sum_{i, j}\left(a_{i j}\right)_{v}^{\prime \prime}\left(W_{1}, W_{2}\right) d x^{i} d x^{j}
$$

But

$$
g_{v}(U, V)=g_{v}\left(U, V_{M}\right)=g_{v}\left(U_{M}, V\right)=g_{v}\left(U_{M}, V_{M}\right),
$$

and the same thing for $g_{v}^{W}$ and $g_{v}^{W_{1}, W_{2}}$, then (12) becomes

$$
\begin{aligned}
g_{\nu}^{W_{1}, W_{2}}(U, V) & =g_{\Psi(\nu)}^{\Psi_{\nu}^{\prime \prime}\left(W_{1}, W_{2}\right)}\left(\Psi_{\nu}^{\prime}(U), \Psi_{\nu}^{\prime}(V)\right)+g_{\Psi(\nu)}^{\Psi_{\nu}^{\prime}\left(W_{1}\right), \Psi_{\nu}^{\prime}\left(W_{2}\right)}\left(\Psi_{\nu}^{\prime}(U), \Psi_{\nu}^{\prime}(V)\right) \\
& +g_{\Psi(\nu)}^{\Psi_{\nu}^{\prime}\left(W_{1}\right)}\left(\phi_{\nu}^{\prime \prime}\left(U, W_{2}\right), \Psi_{\nu}^{\prime}(V)\right)+g_{\Psi(\nu)}^{\Psi_{\nu}^{\prime}\left(W_{1}\right)}\left(\Psi_{\nu}^{\prime}(U), \phi_{\nu}^{\prime \prime}\left(V, W_{2}\right)\right) \\
& +g_{\Psi(\nu)}^{\Psi_{\nu}^{\prime}\left(W_{2}\right)}\left(\phi_{\nu}^{\prime \prime}\left(U, W_{1}\right), \Psi_{\nu}^{\prime}(V)\right)+g_{\Psi(\nu)}^{\Psi_{\nu}^{\prime}\left(W_{2}\right)}\left(\Psi_{\nu}^{\prime}(U), \phi_{\nu}^{\prime \prime}\left(V, W_{1}\right)\right) \\
& +g_{\Psi(\nu)}\left(\phi_{\nu}^{\prime \prime}\left(U, W_{1}\right), \phi_{\nu}^{\prime \prime}\left(V, W_{2}\right)\right)+g_{\Psi(\nu)}^{\prime \prime}\left(\phi_{\nu}^{\prime \prime}\left(U, W_{2}\right), \phi_{\nu}^{\prime \prime}\left(V, W_{1}\right)\right) \\
& +g_{\Psi(\nu)}\left(\phi_{\nu}^{\prime \prime \prime}\left(U, W_{1}, W_{2}\right), \Psi_{\nu}^{\prime}(V)\right)+g_{\Psi(\nu)}\left(\Psi_{\nu}^{\prime}(U), \phi_{\nu}^{\prime \prime \prime}\left(V, W_{1}, W_{2}\right)\right)
\end{aligned}
$$

Since $\Psi$ has a trivial 1-jet at $\nu, \Psi(\nu)=(p, r), \Psi_{\nu}^{\prime}=I d$ and $\phi_{\nu}^{\prime \prime}=0$ (Step 1), and so

$$
\begin{aligned}
& g_{(p, r)}\left(\phi_{(p, r)}^{\prime \prime \prime}\left(U, W_{1}, W_{2}\right), V\right)+g_{(p, r)}\left(U, \phi_{(p, r)}^{\prime \prime \prime}\left(V, W_{1}, W_{2}\right)\right) \\
&=-g_{(p, r)}^{\Psi_{(p, r)}^{\prime \prime}}\left(W_{1}, W_{2}\right) \\
&
\end{aligned}
$$




$$
\begin{aligned}
\underset{g_{(p, r)}}{\Psi_{(p, r)}^{\prime \prime}\left(W_{1}, W_{2}\right)}(U, V) & =\sum_{i, j}\left(a_{i j}\right)_{(p, r)}^{\prime}\left(\Psi_{(p, r)}^{\prime \prime}\left(W_{1}, W_{2}\right)\right)(U)_{i}(V)_{j} \\
& =\sum_{i, j}\left(\left(\mathbf{D} a_{i j}\right)_{(p, r)}\left(\phi_{(p, r)}^{\prime \prime}\left(W_{1}, W_{2}\right)\right)+\delta_{(p, r)}^{\prime \prime}\left(W_{1}, W_{2}\right) \partial_{(p, r)} a_{i j}\right)(U)_{i}(V)_{j} \\
& =\sum_{i, j} \delta_{(p, r)}^{\prime \prime}\left(W_{1}, W_{2}\right) \partial_{(p, r)} a_{i j}(U)_{i}(V)_{j} \\
& =\delta_{(p, r)}^{\prime \prime}\left(W_{1}, W_{2}\right) \partial_{(p, r)} g(U, V)
\end{aligned}
$$

where

$$
\partial_{(p, r)} g(U, V)=\sum_{i, j} \partial_{(p, r)} a_{i j}(U)_{i}(V)_{j}
$$

Thus (14) gives

$$
\begin{aligned}
g_{(p, r)}\left(\phi_{(p, r)}^{\prime \prime \prime}\left(U, W_{1}, W_{2}\right), V\right) & +g_{(p, r)}\left(U, \phi_{(p, r)}^{\prime \prime \prime}\left(V, W_{1}, W_{2}\right)\right) \\
& =-\delta_{(p, r)}^{\prime \prime}\left(W_{1}, W_{2}\right) \partial_{(p, r)} g(U, V)
\end{aligned}
$$

Finally, apply the Generalized Braid Lemma to $J=g_{(p, r)}, K=\delta_{(p, r)}^{\prime \prime}, J^{\prime}=-\partial_{(p, r)} g$ and $A=\phi_{(p, r)}^{\prime \prime \prime}$ to get $\phi_{(p, r)}^{\prime \prime \prime}=0$.

6.4. Step 3: the $\delta$-part, end of proof of Theorem 2.2. Let $\mathcal{V}$ be a generic lightlike manifold. If $\Psi=(\phi, \delta)$ is a 3-isometry at $(p, r)$ with a trivial 1-jet at $(p, r)$ then $\Psi$ has a trivial 2-jet at $(p, r)$.

Proof. By step 2 we have $\phi_{(p, r)}^{\prime \prime \prime}=0$, so (19) gives

$$
\delta_{(p, r)}^{\prime \prime}\left(W_{1}, W_{2}\right) \sum_{i, j} \partial_{(p, r)} a_{i j}(U)_{i}(V)_{j}=0
$$

Remember that $g$ is nowhere transversally Riemannian, thus $\delta_{(p, r)}^{\prime \prime}=0$, and hence $\Psi$ has a trivial 2 -jet.

Thus, Theorem 2.2 in the case $d=1$, that is $(3,1)$-subrigidity of generic lightlike metrics, is fully proved.

6.5. Proof in the case $d>1$. If $\Psi=(\phi, \delta)$ is isometric up to order $d+2$ at a point $(p, r) \in \mathcal{V}$, then the equality of (8) holds for the derivatives of order $d+1$ at $(x, t)=(p, r)$. A derivation of order $d$ of the left side gives

$$
\left(g_{(x, t)}(U, V)\right)_{(p, r)}^{(d)}\left(W_{1}, W_{2}, \ldots, W_{d}\right)=\sum_{i, j}\left(a_{i j}\right)_{(p, r)}^{(d)}\left(W_{1}, W_{2}, \ldots, W_{d}\right)(U)_{i}(V)_{j}
$$

for all $\left(W_{1}, W_{2}, \ldots, W_{d}\right) \in T_{(p, r)} \mathcal{V}$. On the other hand, taking derivation of the right side of $(8)$ at $(p, r)$, and using the fact that $\Psi$ has a trivial $d$-jet at $(p, r)$, it remains

$$
\begin{aligned}
& \sum_{i, j}\left(a_{i j}\right)_{(p, r)}^{(d)}\left(W_{1}, W_{2}, \ldots, W_{d}\right)(U)_{i}(V)_{j}+\sum_{i, j} a_{i j}(p, r)\left(\phi_{(p, r)}^{(d+1)}\left(U, W_{1}, W_{2}, \ldots, W_{d}\right)\right)_{i}(V)_{j} \\
& +\sum_{i, j} a_{i j}(p, r)(U)_{i}\left(\phi_{(p, r)}^{(d+1)}\left(V, W_{1}, W_{2}, \ldots, W_{d}\right)\right)_{j}
\end{aligned}
$$


Comparing with (16), we get,

$\sum_{i, j} a_{i j}(p, r)\left(\phi_{(p, r)}^{(d+1)}\left(U, W_{1}, W_{2}, \ldots, W_{d}\right)\right)_{i}(V)_{j}+\sum_{i, j} a_{i j}(p, r)(U)_{i}\left(\phi_{(p, r)}^{(d+1)}\left(V, W_{1}, W_{2}, \ldots, W_{d}\right)\right)_{j}=0$

that is,

$$
g_{(p, r)}\left(\phi_{(p, r)}^{(d+1)}\left(U, W_{1}, W_{2}, \ldots, W_{d}\right), V\right)+g_{(p, r)}\left(U, \phi_{(p, r)}^{(d+1)}\left(V, W_{1}, W_{2}, \ldots, W_{d}\right)\right)=0
$$

By the Braid Lemma 4.1 we conclude that $\phi_{(p, r)}^{(d+1)}=0$.

Now, if we derive $(8)(d+1)$-times at $(p, r)$, we get for the left side

$$
\sum_{i, j}\left(a_{i j}\right)_{(p, r)}^{(d+1)}\left(W_{1}, W_{2}, \ldots, W_{d+1}\right)(U)_{i}(V)_{j}
$$

and for the right one

$$
\begin{aligned}
& \sum_{i, j}\left(a_{i j}\right)_{(p, r)}^{(d+1)}\left(W_{1}, W_{2}, \ldots, W_{d+1}\right)(U)_{i}(V)_{j}+\sum_{i, j}\left(a_{i j}\right)_{(p, r)}^{\prime}\left(\Psi_{(p, r)}^{(d+1)}\left(W_{1}, W_{2}, \ldots, W_{d+1}\right)\right)(U)_{i}(V)_{j} \\
& +\sum_{i, j} a_{i j}(p, r)\left(\Psi_{(p, r)}^{(d+2)}\left(U, W_{1}, \ldots, W_{d+1}\right)\right)_{i}(V)_{j} \\
& +\sum_{i, j} a_{i j}(p, r)(U)_{i}\left(\Psi_{(p, r)}^{(d+2)}\left(V, W_{1}, \ldots, W_{d+1}\right)\right)_{j}
\end{aligned}
$$

for any $W_{1}, W_{2}, \ldots, W_{d+1} \in T_{(p, r)} \mathcal{V}$, since $\Psi$ has a trivial $d$-jet at $(p, r)$ and $\phi_{(p, r)}^{(d+1)}=0$.

Writing equality between the two sides gives,

$$
\begin{aligned}
& \sum_{i, j} a_{i j}(p, r)\left(\phi_{(p, r)}^{(d+2)}\left(U, W_{1}, \ldots, W_{d+1}\right)\right)_{i}(V)_{j}+\sum_{i, j} a_{i j}(p, r)(U)_{i}\left(\phi_{(p, r)}^{(d+2)}\left(V, W_{1}, \ldots, W_{d+1}\right)\right)_{j} \\
& =-\sum_{i, j}\left(a_{i j}\right)_{(p, r)}^{\prime}\left(\Psi_{(p, r)}^{(d+1)}\left(W_{1}, W_{2}, \ldots, W_{d+1}\right)\right)(U)_{i}(V)_{j}
\end{aligned}
$$

that is

$$
\begin{aligned}
g_{(p, r)}\left(\phi_{(p, r)}^{(d+2)}\left(U, W_{1}, \ldots, W_{d+1}\right), V\right) & +g_{(p, r)}\left(U, \phi_{(p, r)}^{(d+2)}\left(V, W_{1}, \ldots, W_{d+1}\right)\right) \\
& =-g_{(p, r)}^{\Psi_{(p, r)}^{(d+1)}\left(W_{1}, \ldots, W_{d+1}\right)}(U, V)
\end{aligned}
$$

But

$$
\begin{aligned}
g_{(p, r)}^{\Psi_{(p, r)}^{(d+1)}\left(W_{1}, \ldots, W_{d+1}\right)}(U, V)= & \sum_{i, j}\left(a_{i j}\right)_{(p, r)}^{\prime}\left(\Psi_{(p, r)}^{(d+1)}\left(W_{1}, \ldots, W_{d+1}\right)\right)(U)_{i}(V)_{j} \\
= & \sum_{i, j}\left(\mathbf{D} a_{i j}\right)_{(p, r)}\left(\phi_{(p, r)}^{(d+1)}\left(W_{1}, \ldots, W_{d+1}\right)\right)(U)_{i}(V)_{j} \\
& +\sum_{i, j} \delta_{(p, r)}^{(d+1)}\left(W_{1}, \ldots, W_{d+1}\right) \partial_{(p, r)} a_{i j}(U)_{i}(V)_{j} \\
= & \sum_{i, j} \delta_{(p, r)}^{(d+1)}\left(W_{1}, \ldots, W_{d+1}\right) \partial_{(p, r)} a_{i j}(U)_{i}(V)_{j} \\
= & \delta_{(p, r)}^{(d+1)}\left(W_{1}, \ldots, W_{d+1}\right) \partial_{(p, r)} g(U, V)
\end{aligned}
$$


Thus (18) becomes

$$
\begin{aligned}
& g_{(p, r)}\left(\phi_{(p, r)}^{(d+2)}\left(U, W_{1}, \ldots, W_{d+1}\right), V\right)+g_{(p, r)}\left(U, \phi_{(p, r)}^{(d+2)}\left(V, W_{1}, \ldots, W_{d+1}\right)\right) \\
= & -\delta_{(p, r)}^{(d+1)}\left(W_{1}, \ldots, W_{d+1}\right) \partial_{(p, r)} g(U, V)
\end{aligned}
$$

Applying the Generalized Braid Lemma 4.2 to $J=g_{(p, r)}, K=\delta_{(p, r)}^{(d+1)}\left(., ., W_{3}, \ldots, W_{d+1}\right)$, $J^{\prime}=-\partial_{(p, r)} g$ and $A=\phi_{(p, r)}^{(d+2)}\left(., ., ., W_{3}, \ldots, W_{d+1}\right)$, we get $\phi_{(p, r)}^{(d+2)}=0$ and

$$
-\delta_{(p, r)}^{(d+1)}\left(W_{1}, \ldots, W_{d+1}\right) \partial_{(p, r)} g(U, V)=0
$$

which means that $\delta_{(p, r)}^{(d+1)}=0$ since $g$ is nowhere transversally Riemannian. Therefore $\Psi$ has a trivial $(d+1)$-jet. This completes the proof of Theorem 2.2.

\section{REFERENCES}

[1] Ballmann W. Geometric structures, people.mpim-bonn.mpg.de/hwbllmnn/notes.html Lecture notes page.

[2] Bekkara, E.; Frances, C. and Zeghib, A. On lightlike geometry: isometric actions, and rigidity aspects. C. R. Math. Acad. Sci. Paris 343 (2006), no. 5, 317-321.

[3] Bekkara, S.; Zeghib, A. Singular Riemannian metrics, sub-rigidity versus rigidity. Math. Res. Lett. 18 (2011), no. 6, 1203-1214.

[4] Bekkara, S.; Zeghib, A. On automorphism groups of generalized conformal structures. In preparation.

[5] Benoist, Y. Orbites des structures rigides (d'après M. Gromov). Feuilletages et systèmes intégrables (Montpellier, 1995), 1-17, Progr. Math., 145, Birkhäuser Boston, Boston, MA, (1997).

[6] Berger M. Geometry I. Springer, Corrected Fourth Printing (2009).

[7] Candel, A.; Quiroga-Barranco, R. Rigid and finite type geometric structures. Geom. Dedicata 106 (2004), 123-143.

[8] D'Ambra, G.; Gromov, M. Lectures on transformation groups: geometry and dynamics. Surveys in differential geometry (Cambridge, MA, 1990), 19-111, Lehigh Univ., Bethlehem, PA, (1991).

[9] Gromov, M. Partial differential relations. Springer-Verlag, Berlin (1986).

[10] Frances, C. Une démonstration du théorème de Liouville en géométrie conforme. Enseignement Mathématique. (2) 49 (2003), no. 1-2, 95-100.

[11] Frances, C. Sur le groupe d'automorphismes des géométries paraboliques de rang 1. Ann. Sci. École Norm. Sup. (4) 40 (2007), no. 5, 741-764.

[12] Gromov, M. Rigid transformations groups. Géométrie différentielle (Paris, 1986), 65-139, Travaux en Cours, 33, Hermann, Paris, (1988).

[13] Katok, A and Hasselblatt, B. Introduction to the modern theory of dynamical systems. Cambridge University Press, Cambridge, (1995).

[14] Kobayashi, S. Transformation groups in differential geometry. Reprint of the 1972 edition. Classics in mathematics. Springer-Verlag, Berlin; (1995).

[15] Spivak, M. A comprehensive introduction to differential geometry, Volume 3, Publish or Perish (1999).

[16] Sternberg S. Lectures on differential geometry. Prentice-Hall Inc. Englewood Cliffs; N.J. (1964).

[17] Zeghib A. On Gromov's theory of rigid transformation groups: A dual approach. Ergodic Theory Dynam. Systems 20 (2000), no. 3, 935-946. 
S. Bekkara, Department of Mathematics, USt-Oran, Algeria

E-mail address: samir.bekkara@gmail.com

A. Zeghib, CNRS, UMPA, ENS-Lyon, France

E-mail address: abdelghani.zeghib@ens-lyon.fr http://www. umpa.ens-lyon.fr/ zeghib/ 